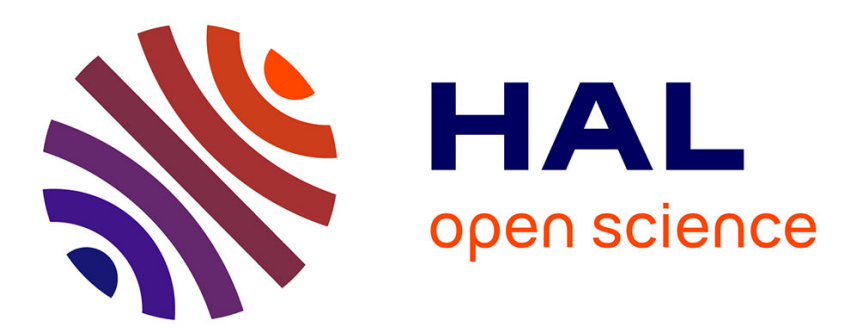

\title{
Managing a multi battery energy storage system of a telecommunications company in order to reduce the total energy cost
}

Isaias Faria Silva, Cédric Bentz, Mustapha Bouhtou, Matthieu Chardy, Safia Kedad-Sidhoum

\section{To cite this version:}

Isaias Faria Silva, Cédric Bentz, Mustapha Bouhtou, Matthieu Chardy, Safia Kedad-Sidhoum. Managing a multi battery energy storage system of a telecommunications company in order to reduce the total energy cost. International Journal of Smart Grid and Clean Energy, 2022, 10.12720/sgce.11.1.113. halshs-03472030

\section{HAL Id: halshs-03472030 \\ https://shs.hal.science/halshs-03472030}

Submitted on 9 Dec 2021

HAL is a multi-disciplinary open access archive for the deposit and dissemination of scientific research documents, whether they are published or not. The documents may come from teaching and research institutions in France or abroad, or from public or private research centers.
L'archive ouverte pluridisciplinaire HAL, est destinée au dépôt et à la diffusion de documents scientifiques de niveau recherche, publiés ou non, émanant des établissements d'enseignement et de recherche français ou étrangers, des laboratoires publics ou privés. 


\title{
Managing a multi battery energy storage system of a telecommunications company in order to reduce the total energy cost
}

\author{
Isaías F. Silva a,b, Cédric Bentz ${ }^{\text {b }}$, Mustapha Bouhtou ${ }^{\text {a }}$, Matthieu Chardy a, Safia \\ Kedad-Sidhoum ${ }^{\mathrm{b}}$ \\ ${ }^{a}$ Orange Labs, Chatîllon, France \\ ${ }^{b}$ Cédric-CNAM, Paris, France
}

\begin{abstract}
The use of batteries as a backup in case of power outages is a common practice adopted by telecommunications companies that need to keep their services always active. Besides, those batteries can also be used for other purposes such as participating in the energy market in order to reduce the electricity bill, as long as the safety usage rules are respected. In this context, batteries can be used when the energy costs more and recharged when the energy costs less, which is known as the demand-response mechanism. Our focus in this work is to optimize the use of batteries installed for backup to participate in the demand-response mechanism, in order to reduce the total energy cost for the company. We formally state the related optimization problem and propose two solving approaches to address it: a mixed-integer program and a heuristic to solve large instances. Simulations based on real data of a French telecommunications operator prove the relevance of using batteries to reduce the energy cost for the company by participating in the demandresponse mechanism. The proposed heuristic proves to be economically relevant and computationally efficient, being a good alternative to a mixed-integer program for large-scale problems.
\end{abstract}

Keywords: Multi battery energy storage system, demand-response mechanism, optimization, mixed-integer program, heuristic

\section{Introduction}

The electrical energy market has widely evolved over the decades, especially with the emergence of the smart-grids. Dynamic battery storage systems and renewable energy sources together with new information and communication technologies allow those of consumption and production agents to participate collaboratively in the energy markets $[1,2]$.

In this context, batteries are used in different ways. In telecommunications companies, it is common to use batteries as a backup in the case of power failures, since such companies provide critical services and must therefore keep their network always active [3]. These batteries are used in conjunction with antennas and other equipment, and, in order to ensure that they are always operating in the case of a power failure, strict safety management rules must be considered. Moreover, a study was done at the French telecommunications company Orange by Marquet et al. [4], and addressed the use of batteries in telecommunications systems to reduce the use of fuel, the OPerational EXpenditure (OPEX) of energy remote plants and, if possible, to remove the diesel engines that are installed in remote stations. In addition, the company may use those batteries to participate in the energy market as a coordinated battery storage system, ensuring that the grid is properly reliable as long as the safety use rules are respected.

\footnotetext{
* Manuscript received May 21, 2021; revised October 14, 2021.

Corresponding authors. E-mail address: isaias.faria@orange.com

doi: $10.12720 /$ sgce.11.1.1-13
} 


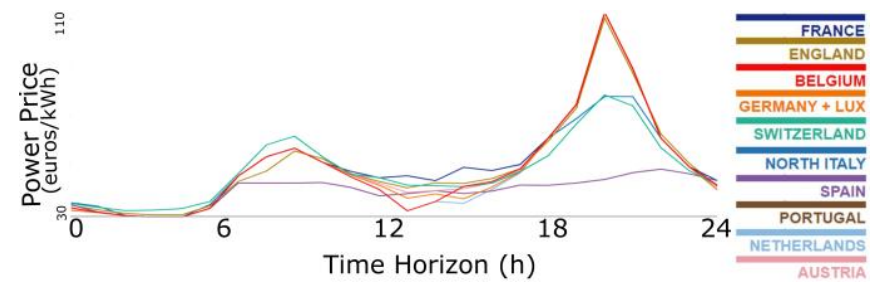

Fig. 1. Energy spot-prices - September, 14th, 2020 [10].

Indeed, as the energy price changes over time, batteries may be used to avoid buying energy when the price is high, which is known as the demand-response mechanism [5]. The batteries will then be recharged when the energy price is low, generating savings [6]. The energy prices over a day are defined by energy production and demand, and must be paid to buy energy from the market. Such an energy market is known as Retail Market, and the demand-response mechanism has been widely studied over the last decade $[7,8,9]$. This mechanism can be defined as the changes in electric usage by end-use customers from their normal consumption patterns in response to changes in the energy prices over time. Fig. 1 illustrates the variability of energy prices on September, 14th, 2020 in Europe.

Daryanian et al. used in [5] a single battery to reduce the electricity bill by exploiting the variation of the energy prices. In this study, a battery is used in peak-time periods, where the energy costs more, and recharged in periods where the energy is cheaper. Several later studies explore the demand-response mechanism in different usage scenarios and with various solving approaches $[8,11,12,13]$. Among them, linear programming is frequently used as a solution method to reduce the energy cost by optimizing the battery use, such as in $[11,12,14,15]$. As an example, Hoke et al. study the use of a battery to minimize the cost of operating a microgrid while meeting resource constraints from conventional generators, solar panels, and wind turbines [11]. Moreover, Good et al. [12] treat the uncertainty in power demand, renewable energy generation, and prices, through the use of a linear program with a robust strategy.

Another challenge is the use of multiple Battery Energy Storage Systems (BESS), requiring more efficient control strategies for optimal management. In this context, recent studies propose different methods to treat the dimensionality efficiently $[16,17,18]$. Babazadeh et al. [16] propose a multiple battery management system with different types of battery focusing on the minimization of the total system cost and considering the impact of the usage on the lifetime of the batteries. In the same vein, Zhu et al. [17] present an adaptive dynamic program and Fan et al. [18] a convex quadratic optimization problem to optimize a multiple battery storage system properly. In our case, we consider a multiple BESS for which real engineering usage rules must be respected for any battery use for safety reasons.

In this study, we consider the problem of optimizing the total energy costs using batteries installed for backup to participate in the retail market, using proper battery management. Our goal is thus to reduce the total energy OPEX for the company using proper battery management. In this context, Faria et al. [19] consider single battery management to participate in the retail market. They also consider the use of such a battery in the curtailment market, acting as a direct agent in the grid stabilization. In our case, we aim to use multiple batteries installed for backup to participate only in the retail market, while considering strict safety usage rules.

Formally, the problem under study is the Optimization of a Multi-Battery Storage system participating in the Retail market in a demand-response mechanism (referred to as OMBSR), in order to reduce the total energy cost for the company. The main issue for such a company is to respect the market rules and the battery safety usage rules while minimizing the net total energy cost. Moreover, the decision version of such an optimization problem is NP-Complete, which follows from a reduction from the 3-Partition problem (not detailed here).

Our first contribution is the modeling of the market rules and battery safety usage rules through a mixed-integer linear program (MILP) for the problem under study, whose optimal solution provides the best way to generate savings for the company, i.e., to reduce the total energy cost. Our second contribution is a heuristic, which can be used as a workaround method for solving large-scale instances. Our third 
contribution is the validation of our algorithms on instances based on real datasets from the mobile $4 \mathrm{G}$ network of the French telecommunications operator Orange. Firstly, we show that using batteries installed for backup to participate in the retail market yields savings for the company. Secondly, we investigate the algorithmic performance and the economic relevance for instances covering different parameter ranges and sizes.

This paper is organized as follows. In Section 2, we formally define the problem and the hypotheses considered. Then, we present a mathematical programming model for the problem in Section 3, and a heuristic in Section 4. Numerical experiments are provided in Section 5. In Section 6, we conclude and give some perspectives for future research.

\section{Problem Statement}

We consider the deterministic framework with a single telecommunication site that we now formally describe. Let us consider a site with a power demand $W_{t}$, given in $\mathrm{kW}$, at each time period $t$ of discrete a horizon $T$ of $T$ equally-sized time periods of duration $\Delta$ minutes $(T=\{1, \ldots, T\})$.

The cost (given in monetary units) for purchasing one unit of energy power at time period $t$ is denoted by $E_{t}$. This cost is fixed by the distributor, as is the maximum amount of power $P^{\max }$, given in $\mathrm{kW}$, that can be bought at any time period.

With respect to the battery assets, a site is equipped with a set $\mathcal{B}$ of batteries. For safety reason, a minimum amount of energy $B_{b}^{\min }$, given in $\mathrm{kWh}$, must always remain in each battery $b \in \mathcal{B}$. In addition, to improve its lifespan, and for network security purposes, each battery $b \in \mathcal{B}$ must be immediately recharged after each use, up to its maximum energy capacity, denoted by $B_{b}^{\max }$ and given in $\mathrm{kWh}$, with a constant power rate $P_{B_{b}}$ given in $\mathrm{kW}$. Note that the battery recharge process is not linear and depends on factors such as the depth of discharge, and the battery power level. However, a linearization of the battery power level during the recharging process is commonly considered. Moreover, a minimum power discharge per time period, denoted by $D_{b}^{\text {min }}$ and given in $\mathrm{kW}$, is imposed when a battery $b$ is in discharge mode. Moreover, each battery has a maximal power rate, denoted by $D_{b}^{\max }$ and given in $\mathrm{kW}$, that it can release at each time period due to current and voltage limitations. Note that $D_{b}^{\min } \in\left[0, D_{b}^{\max }\right]$, and that the power demand $W_{t}$ is higher than $D_{b}^{\min }$ at all time periods $t$ over the horizon. To simplify the model writing, and since the same time discretization $\Delta$ is considered over the time horizon, we can treat the amount of energy in each battery as its power capacity. Each battery must also be fully charged at the beginning and at the end of the planning horizon.

Concerning the battery life cycle, the number of times that each battery can be used is limited. In this context, each battery $b$ can be used at most $N_{b}$ times over the time horizon. Recall that our goal is to manage the use of the batteries while respecting both the usage and the energy retail market rules, and keeping the network safe at optimal cost. The total amount of energy savings that can be obtained is provided by the difference between the energy prices during a battery use and its recharge. The amount of energy not bought during the battery use is equal to the battery discharge. Furthermore, we consider only one energy supplier without renewable energy sources. The batteries are ready for use, and hence no installation cost is considered.

The problem stated above is referred to as OMBSR in the following, and any of its instances is fully described by the following parameters (some of which are vectors or sets): $W, \Delta, E, P^{\max }, \mathcal{B}, B^{\min }, B^{\max }, P_{B}, D^{\min }, D^{\max }, N$.

\section{OMBSR Mixed Integer Nonlinear Programming}

The formulation described in this section that models OMBSR as a mixed-integer nonlinear program, will be referred to as $(M I N L P)$.

\section{Model Variables}

Firstly, a solution is determined by the values of the following variables:

- $\quad x_{b, t} \in\left[B_{b}^{\min }, B_{b}^{\max }\right], \forall b \in \mathcal{B}, \forall t \in T$ : equal to the power capacity (in $\mathrm{kW}$ ) of each battery $b$ 
at the beginning of each time period $t$. We consider such an amount as a power capacity, given in $\mathrm{KW}$, because the amount of energy of a battery is treated as its power capacity. An additional variable $x_{b, T+1}$ represents the power capacity at the end of the last time period.

The following additional variables are used to control the state of each battery:

- $z_{b, t} \in\{0,1\}, \forall b \in \mathcal{B}, \forall t \in T$ : equal to 1 if the battery $b$ is in discharge mode at time period $t$, and to 0 otherwise;

- $\quad b_{b, t}^{\text {start }} \in\{0,1\}, \forall b \in \mathcal{B}, \forall t \in T$ : equal to 1 if the battery $b$ starts being discharged at time period $t$, and to 0 otherwise.

To model the power bought at each time period $t$, the following variables are used:

- $\quad u_{t}^{\mathrm{D}} \in\left[0, W_{t}\right], \forall t \in T$ : power bought for the demand consumption at time period $t$ (in $\mathrm{kW}$ );

- $u_{b, t}^{\mathrm{B}} \in\left[0, P_{B_{b}}\right], \forall b \in \mathcal{B}, \forall t \in T$ : power bought for the recharge of each battery $b$ at time period $t$ (in $\mathrm{kW})$.

\section{Mixed Integer Nonlinear Programming Formulation}

The objective function is defined as follows:

$$
\min \sum_{t \in T} E_{t}\left(\sum_{b \in \mathcal{B}} u_{b, t}^{\mathrm{B}}+u_{t}^{\mathrm{D}}\right)
$$

The objective function minimizes the total energy cost spent on purchasing energy. A solution is given by the power capacity of the batteries at each time period (the values of the $x_{b, t}$ variables).

The following constraints define the state of each battery at each time period $t$ :

$$
\begin{array}{ll}
x_{b, t}-x_{b, t+1} \leq z_{b, t} D_{b}^{\max } & \forall b \in \mathcal{B}, \forall t \in T \\
-x_{b, t}+x_{b, t+1} \leq P_{B_{b}}\left(1-z_{b, t}\right)-z_{b, t} D_{b}^{\min } & \forall b \in \mathcal{B}, \forall t \in T
\end{array}
$$

Constraints (2) guarantee that, if the power capacity of a battery decreases, then the battery is in discharge mode, i.e., $z_{b, t}=1$. Constraints (3) ensure that, if the power capacity of a battery increases, then this battery cannot be in discharge mode, i.e., $z_{b, t}=0$. Note that, together with Constraints (9) and (10), these constraints ensure that the battery can have the same power capacity during two consecutive time periods only if the battery is fully charged, otherwise a minimal discharge of $D_{b}^{\min }$ (if $z_{b, t}=1$ ) or a recharge of $u_{b, t}^{\mathrm{B}}$ (if $z_{b, t}=0$ ) is imposed. Moreover, Constraints (2) guarantee a maximum power discharge per time period of $D_{b}^{\max }$ when the battery is in discharge mode.

In the same vein, Constraints (4) and (5) ensure that $b_{b, t}^{\text {start }}=1$ if the battery $b$ starts being discharged at time period $t$; otherwise, this variable is free.

$$
\begin{array}{ll}
b_{b, t}^{\text {start }} \geq z_{b, t}-z_{b, t-1} & \forall b \in \mathcal{B}, \forall t \in T \backslash\{1\} \\
b_{b, t}^{\text {start }}=z_{b, 1} & \forall b \in \mathcal{B}
\end{array}
$$

Constraints (6) guarantee that each battery $b$ can start being discharged only if it is fully charged (and hence together with Constraints (7) that the battery starts being recharged immediately after each use, up to its maximum capacity):

$$
B_{b}^{\text {max }} b_{b, t}^{\text {start }} \leq x_{b, t} \quad \forall b \in \mathcal{B}, \forall t \in T
$$

The power purchased in the retail market at each time period $t$ is the sum of the power bought for charging the batteries $\left(\sum_{b \in \mathcal{B}} u_{b, t}^{\mathrm{B}}\right)$ and the power bought for consumption $\left(u_{t}^{\mathrm{D}}\right)$, which is ensured by the following constraints:

$$
\begin{array}{lr}
u_{b, t}^{\mathrm{B}}=\left(1-z_{b, t}\right) \min \left(B_{b}^{\max }-x_{b, t}, P_{B_{b}}, P^{\max }\right) & \forall b \in \mathcal{B}, \forall t \in T \\
\sum_{b \in \mathcal{B}}\left(x_{b, t+1}-x_{b, t}\right)=\sum_{b \in \mathcal{B}} u_{b, t}^{\mathrm{B}}+u_{t}^{\mathrm{D}}-W_{t} & \forall t \in T
\end{array}
$$




$$
\begin{array}{ll}
x_{b, t+1}-x_{b, t} \geq u_{b, t}^{\mathrm{B}}-z_{b, t} D_{b}^{\max } & \forall b \in \mathcal{B}, \forall t \in T \\
x_{b, t+1}-x_{b, t} \leq u_{b, t}^{\mathrm{B}} & \forall b \in \mathcal{B}, \forall t \in T
\end{array}
$$

The power bought for charging each battery is $\min \left(P_{B_{b}}, P^{\max }\right)$ when it is possible to buy energy (i.e., if $z_{b, t}=0$ ), if the capacity of the battery is not exceeded (see Constraints (7)). Note that several batteries can be used at the same time: some of them can be in discharge mode and others recharging. Constraints (9) together with Constraints (10) guarantee that the power bought to recharge a battery is properly routed, as no power exchange is technically allowed between batteries.

Since no losses are considered, the power balance of the batteries is ensured by Constraints (8). Moreover, Constraints (8) impose a maximum accumulated discharge of all batteries at the same time period equal to the power demand $W_{t}$.

The network capacity is guaranteed by Constraints (11).

$$
\sum_{b \in \mathcal{B}} u_{b, t}^{\mathrm{B}}+u_{t}^{\mathrm{D}} \leq P^{\max }
$$

Furthermore, Constraints (12) guarantee that each battery will be used at most $N_{b}$ times over the time horizon, while Constraints (13) express the limit conditions:

$$
\begin{array}{ll}
\sum_{t \in T} b_{b, t}^{\text {start }} \leq N_{b} & \forall b \in \mathcal{B} \\
x_{b, 1}=x_{b, T+1}=B_{b}^{\max } & \forall b \in \mathcal{B}
\end{array}
$$

Finally, the domains of the variables are:

$$
\begin{array}{lr}
u_{t}^{\mathrm{D}} \in\left[0, W_{t}\right] & \forall t \in T \\
u_{b, t}^{\mathrm{B}} \in\left[0, P_{B_{b}}\right], x_{b, t} \in\left[B_{b}^{\text {min }}, B_{b}^{\text {max }}\right], z_{b, t} \in\{0,1\}, b_{b, t}^{\text {start }} \in\{0,1\} & \forall b \in \mathcal{B}, \forall t \in T
\end{array}
$$

The obtained model (1)-(15) is non-linear. However, it can be linearized following the approach proposed by McCormick [20]. The resulting linear model (referred to as $(M I L P)$ ) is provided in Appendix A.

\section{Graph Oriented Heuristic to OMBSR}

This section presents a graph-oriented temporal decomposition heuristic based on:

- The decomposition of each OMBSR instance into sub-instances that are individually solved to optimality;

- The selection of a subset of the solutions obtained for the sub-instances that respects the maximal number of battery uses $N_{b}$, and that yields a solution to the complete OMBSR instance.
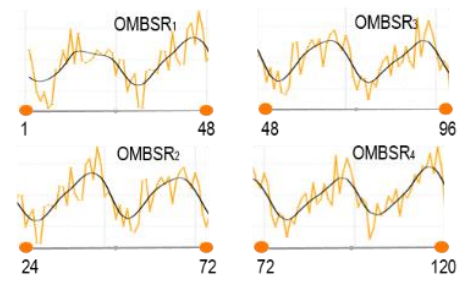

(a)
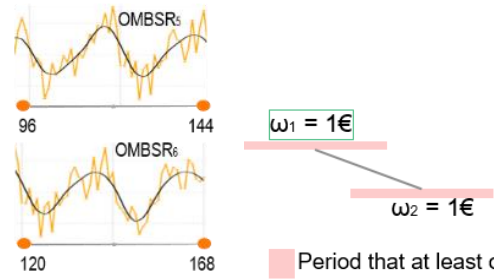

Period that at least one battery is in use in $\mathrm{S}$

(b)

Fig. 2. (a) Decomposition of an OMBSR instance over a week into sub-instances OMBSR 1 to OMBSR 6 , assuming $\delta=$ 48 and $\delta^{\prime}=24$. The curves represent the power demand observed (orange line) and the prediction (black line). (b) Example of a conflict graph associated to the decomposition of the OMBSR instance presented in Fig. 2.a, where $N_{b}=$ $3 \forall b \in \mathcal{B}$, and of the resulting MWIS solution. 
Two integer parameters $\delta$ and $\delta^{\prime}(>0)$ are considered in this heuristic: $\delta$ is the number of time periods in each sub-instance, and $\delta^{\prime}$ is used to define the first time period of each sub-instance. Formally, the heuristic is composed of five steps:

1. Decomposition of the OMBSR instance into sub-instances: We construct $\frac{T}{\delta^{\prime}}-1$ sub-instances OMBSR $_{\mathrm{i}}$ for $i$ in $\left\{1, \ldots,\left(\frac{T}{\delta^{\prime}}-1\right)\right\}$, each one being defined over a reduced time horizon $T_{i}$ of $\delta$ time periods starting at a time period multiple of $\delta^{\prime}$ (i.e., $T_{i}=\left\{\left((i-1) \delta^{\prime}+1\right), \ldots,((i-\right.$ 1) $\delta^{\prime}+\delta$ ) and with at most $N_{b}^{\prime}=\left\lceil\frac{N_{b} \delta}{T}\right\rceil$ battery uses

2. Resolution of $\mathrm{OMBSR}_{\mathrm{i}}$ sub-instances: Considering the MILP formulation for OMBSR based on $T$ and $N_{b}$, we derive the formulation for each $\mathrm{OMBSR}_{\mathrm{i}}$ by considering $T_{i}$ and $N_{b}^{\prime}$ instead. Then, an optimal solution $S_{i}$ for each $\mathrm{OMBSR}_{\mathrm{i}}$ is obtained by solving this formulation with a mixed-integer linear program solver.

3. Construction of a solution conflict graph: A graph $G=(V, E)$ is created, where each node $v_{i}$ in $V$ represents the optimal solution $S_{i}$ of $\mathrm{OMBSR}_{\mathrm{i}}$ found in Step 2, with a weight $\omega_{v_{i}}$ equal to its value. An edge $e=\left(v_{i}, v_{j}\right)$ is added if any battery in the corresponding solution $S_{i}$ is used at a time period $t \in T_{i} \cap T_{j}$ and if any battery (not necessarily the same) is used in the solution $S_{j}$ at the same time period. Note that $G$ is an interval graph.

4. Computation of a multi-criteria Maximal Weight Independent Set (MWIS) of $G$ : We rely on an integer linear program for the MWIS problem on interval graphs, with $|\mathcal{B}|$ additional constraints limiting the number of use of the $b \in \mathcal{B}$ batteries to $N_{b}$ in the selected nodes. The complete formulation, denoted by (MWIS-BAT), is presented in Appendix (C). Then, an optimal solution is obtained by solving it with a standard MILP solver.

5. Construction of a solution for OMBSR: Firstly, the final heuristic solution to the initial OMBSR instance is equal to a standard solution where no battery is used. Then, for each node $v_{i}$ of the solution provided in Step 4 by solving $(M W I S-B A T)$, we replace the standard solution during the period $T_{i}$ by the solution $S_{i}$.

For the sake of clarity, we illustrate in Figures 2.a and 2.b the steps of our generic heuristic on an illustrative OMBSR instance over a week where $N_{b}$ for all $b \in \mathcal{B}$, and for the following choice of parameters: $\delta=48$ and $\delta^{\prime}=24$. A heuristic solution to this OMBSR instance is thus obtained by considering the battery usage in the solutions $S_{1}, S_{4}$ and $S_{6}$ found for sub-instances OMBSR $1, \mathrm{OMBSR}_{4}$ and $\mathrm{OMBSR}_{6}$, respectively.

\section{Numerical Experiments}

In order to assess the efficiency and relevance of our solving approaches for optimizing the savings associated with the demand-response mechanism, we performed some numerical experiments on real-life instances. Several sites with different consumption profiles and BESS are considered, generated from internal data of the French telecommunications operator Orange. The energy costs considered are taken from public historic data of the French retail market.

Two solving approaches are considered. Firstly, the default branch and bound algorithm of the commercial solver CPLEX performed on the formulation (MILP) that will be denoted by MILP. Secondly, the general heuristic presented in Section 4 parameterized by $\delta=48$ and $\delta^{\prime}=24$, that will be denoted by HEU. The arguments (essentially the periodical structure of energy costs and demand of our data) for choosing these parameters are given in Appendix B.

The numerical experiments are organized as follows. Firstly, in Section 5.1, we describe the instances and the tests setting used in our tests. Then, in Section 5.2 we present the results of OMBSR instances solved with MILP and HEU. The impact of the number of batteries as well as the number of time periods is analyzed in the same section. 


\subsection{Instances description}

We based our testbed on 50 urban and rural sites from the mobile $4 \mathrm{G}$ network of the French telecommunications operator Orange. The power consumption and the mean, or average value, of the power demand over the horizon, denoted by $\bar{W}=\frac{\Sigma_{t \in T} W_{t}}{T}$, is also given. Each site is equipped with at most 5 batteries, whose main properties are provided in what follows. The autonomy of the batteries varies between 20 and 60 hours. Besides, two types of batteries are installed: GEL and AGM, the recharge power rate $P_{B_{b}}$ being dependent of each type:

1. $P_{B_{b}}=1.95 \%$ of $B_{b}^{\max }$ for GEL batteries;

2. $P_{B_{b}}=3.34 \%$ of $B_{b}^{\max }$ for AGM batteries.

In addition, the minimal power discharge $D_{b}^{\min }$ is $10 \%$ of $D_{b}^{\max }$. Finally, the value of $B_{b}^{\min }$ is $50 \%$ of the battery power capacity, and each battery cannot be used more than 144 times over a year. More precisely, the value of $N_{b}$ is computed as $\left\lceil\frac{3 * T}{7 * 24}\right\rceil$. Concerning the data related to the distributor, we consider the unit cost of energy from the French distributor EDF, publicly available in [21]. Besides, the maximum amount of power that can be purchased per time period $P^{\max }$ is established in a contract. In our tests, to guarantee that the value of $P^{\max }$ is greater than the power demand $W_{t}$ at any time period $t \in T$, we set such a value to $\overline{3 W}$.

Moreover, we assume time horizons of $\{1,2,3,4,8,12,16,20,24\}$ weeks with time discretization $\Delta=60$ minutes. The input values of the power demand, unit cost of energy, and reward over the time horizon, are taken as average values observed. Our tests were performed on 450 instances.

All tests were performed on a server computer with 4GB of RAM and 1 Intel Xeon $2.2 \mathrm{GHz} \mathrm{CPU}$. The method used to solve the (MILP) formulation is the branch-and-bound implemented in CPLEX 12.9, with default settings. The running time is limited to 30 minutes for each instance.

\subsection{Algorithmic results and economic analysis}

Numerical results are displayed in Table 1, both for the solving methods HEU and MILP. In this table, each row stores the average of the results for 10 instances, grouped by the number of batteries installed $"|\mathcal{B}|$ " in the site and by the number of time periods. Column "Stand Cost" corresponds to the standard cost, i.e., the cost when no battery is used, equal to $\sum_{t \in T} E_{t} W_{t}$. Columns " $\bar{W}$ " and "P $P_{B}$ " report the mean of $\bar{W}$ and $P_{B}$, respectively. Besides, the solving time, given in seconds, and the reduction in the total energy cost, given in \%, are provided in columns "CPU" and "savings", respectively, for MILP and HEU. Concerning the results obtained by MILP, column "gap" reports the optimality gap, i.e., the gap between the best integer solution obtained by CPLEX and the best lower bound computed. Furthermore, the row "mean all weeks" stores the average of the properties and of the solving results for all instances with the same number of batteries installed.

Algorithmic Results We begin by focusing on the algorithmic results and observe a significant impact of the number of time periods and number of batteries installed on the performance of both algorithms.

Concerning MILP, optimal values are obtained only for instances where sites have a single battery installed managed over a week, corresponding to $2.5 \%$ of all the tested instances. For all other instances, no optimality guarantee is observed within the CPU time limit. Moreover, the optimality gap observed is significant, varying from $36 \%$ on average for instances where the site is equipped with 2 batteries managed over 2 weeks, up to $30000 \%$ on average for big instances where the site is equipped with 5 batteries managed over 6 months. However, the best solution found gives a reduction in the energy bill (2\% on average) even for the instances with no optimality guarantee. Furthermore, the number of variables and constraints grow linearly with the number of time periods. Even for instances with a single battery installed, MILP cannot reach an optimality guarantee within the CPU time limit for instances with a battery managed over 2 weeks or more.

Concerning HEU, 86.4\% of all instances are solved in less than 30 minutes. We also observe that the number of batteries installed and the number of time periods have an impact on the solving time. Instances 
with 4 or 5 batteries managed over 3 weeks or more require more computational effort due to the large number of sub-instances to be solved. In some cases, more than two hours were needed to solve instances with HEU.

Table 1. MILP and HEU results.

\begin{tabular}{|c|c|c|c|c|c|c|c|c|c|}
\hline \multicolumn{5}{|c|}{ Instances } & \multicolumn{3}{|c|}{ MILP } & \multicolumn{2}{|c|}{$\mathrm{HEU}$} \\
\hline$|\mathcal{B}|$ & \begin{tabular}{|l|}
$\begin{array}{c}\text { Number } \\
\text { of Weeks }\end{array}$ \\
\end{tabular} & $\begin{array}{c}\text { Stand Cost } \\
(€)\end{array}$ & $\begin{array}{c}\bar{W} \\
(\mathrm{~kW})\end{array}$ & \begin{tabular}{|c|}
$P_{B}$ \\
$(\mathrm{~kW})$
\end{tabular} & $\begin{array}{c}\text { savings } \\
(\%)\end{array}$ & $\begin{array}{c}\text { CPU } \\
\text { (s) }\end{array}$ & $\begin{array}{l}\text { gap } \\
(\%)\end{array}$ & $\begin{array}{c}\text { savings } \\
(\%)\end{array}$ & $\begin{array}{l}\text { CPU } \\
\text { (s) }\end{array}$ \\
\hline \multirow{10}{*}{1} & $\begin{array}{l}\text { mean all } \\
\text { weeks }\end{array}$ & 6350 & 3,74 & 2,30 & 2,43 & 1638 & 53,28 & 2,23 & 12 \\
\hline & 1 & 635 & 3,74 & 2,30 & 2,45 & 341 & 0,00 & 2,19 & 1 \\
\hline & 2 & 1270 & 3,74 & 2,30 & 2,48 & 1800 & 36,32 & 2,21 & 2 \\
\hline & 3 & 1905 & 3,74 & 2,30 & 2,48 & 1800 & 47,24 & 2,21 & 3 \\
\hline & 4 & 2540 & 3,74 & 2,30 & 2,45 & 1800 & 52,93 & 2,22 & 5 \\
\hline & 8 & 5077 & 3,74 & 2,30 & 2,41 & 1800 & 63,49 & 2,26 & 9 \\
\hline & 12 & 7619 & 3,74 & 2,30 & 2,42 & 1800 & 66,08 & 2,24 & 14 \\
\hline & 16 & 10159 & 3,74 & 2,30 & 2,42 & 1800 & 68,24 & 2,24 & 19 \\
\hline & 20 & 12700 & 3,74 & 2,30 & 2,41 & 1800 & 69,79 & 2,24 & 24 \\
\hline & 24 & 15242 & 3,74 & 2,30 & 2,35 & \begin{tabular}{|l|l|}
1800 \\
\end{tabular} & 75,27 & 2,23 & 29 \\
\hline \multirow{10}{*}{2} & $\begin{array}{l}\text { mean all } \\
\text { weeks }\end{array}$ & 5846 & 3,44 & 2,10 & 2,22 & 1800 & 89,73 & 2,40 & 45 \\
\hline & 1 & 585 & 3,45 & 2,10 & 2,50 & 1800 & 33,79 & 2,44 & 4 \\
\hline & 2 & 1170 & 3,45 & 2,10 & 2,51 & 1800 & 52,81 & 2,39 & 9 \\
\hline & 3 & 1753 & 3,44 & 2,10 & 2,45 & 1800 & 65,03 & \begin{tabular}{|l|}
2,39 \\
\end{tabular} & 13 \\
\hline & 4 & 2337 & 3,44 & 2,10 & 2,43 & 1800 & 68,43 & 2,40 & 18 \\
\hline & 8 & 4674 & 3,44 & 2,10 & 2,39 & 1800 & 76,17 & 2,40 & 37 \\
\hline & 12 & 7014 & 3,44 & 2,10 & 2,18 & 1800 & 96,89 & \begin{tabular}{|l|}
2,39 \\
\end{tabular} & 55 \\
\hline & 16 & 9354 & 3,44 & 2,10 & 1,92 & 1800 & 125,31 & 2,39 & 73 \\
\hline & 20 & 11692 & 3,44 & 2,10 & 1,89 & 1800 & 134,67 & 2,39 & 90 \\
\hline & 24 & 14034 & 3,44 & 2,10 & 1,76 & 1800 & 154,48 & 2,39 & 107 \\
\hline \multirow{10}{*}{3} & $\begin{array}{l}\text { mean all } \\
\text { weeks }\end{array}$ & 5850 & 3,45 & 2,10 & 1,95 & 1800 & 117,78 & 2,35 & 247 \\
\hline & 1 & 585 & 3,45 & 2,10 & 2,41 & 1800 & 41,73 & 2,36 & 25 \\
\hline & 2 & 1169 & 3,44 & 2,10 & 2,31 & 1800 & 64,62 & 2,36 & 49 \\
\hline & 3 & 1754 & 3,44 & 2,10 & 2,31 & 1800 & 70,21 & 2,36 & 75 \\
\hline & 4 & 2338 & 3,44 & 2,10 & 2,31 & 1800 & 71,93 & 2,35 & 100 \\
\hline & 8 & 4680 & 3,45 & 2,10 & 2,07 & 1800 & 97,80 & 2,34 & 198 \\
\hline & 12 & 7022 & 3,45 & 2,10 & 1,82 & 1800 & 129,12 & 2,35 & 297 \\
\hline & 16 & 9362 & 3,45 & 2,10 & 1,53 & 1800 & 171,82 & 2,35 & 395 \\
\hline & 20 & 11702 & 3,45 & 2,10 & 1,40 & 1800 & 197,84 & 2,35 & \begin{tabular}{|l|l|}
497 \\
\end{tabular} \\
\hline & 24 & 14039 & 3,45 & 2,10 & 1,34 & 1800 & 214,90 & 2,35 & 590 \\
\hline \multirow{10}{*}{4} & $\begin{array}{l}\text { mean all } \\
\text { weeks }\end{array}$ & 7131 & 4,20 & 2,27 & 1,74 & 1800 & 2293,01 & 2,29 & 1522 \\
\hline & 1 & 713 & 4,20 & 2,27 & 2,30 & 1800 & 49,89 & 2,29 & 154 \\
\hline & 2 & 1426 & 4,20 & 2,27 & 2,26 & 1800 & 68,22 & 2,29 & 301 \\
\hline & 3 & 2140 & 4,20 & 2,27 & 2,26 & 1800 & 72,43 & 2,29 & 453 \\
\hline & 4 & 2852 & 4,20 & 2,27 & 2,22 & 1800 & 78,38 & 2,29 & 609 \\
\hline & 8 & 5706 & 4,20 & 2,27 & 1,86 & 1800 & 120,73 & 2,28 & 1267 \\
\hline & 12 & 8559 & 4,20 & 2,27 & 1,56 & 1800 & 165,05 & 2,29 & 1814 \\
\hline & 16 & 11412 & 4,20 & 2,27 & 1,42 & 1800 & 190,82 & 2,29 & 2408 \\
\hline & 20 & 14260 & 4,20 & 2,27 & 0,86 & 1800 & 9037,24 & 2,29 & 3018 \\
\hline & 24 & 17114 & 4,20 & 2,27 & 0,88 & 1800 & 10854,35 & 2,29 & 3671 \\
\hline \multirow{10}{*}{5} & $\begin{array}{l}\text { mean all } \\
\text { weeks }\end{array}$ & 5091 & 3,00 & 2,35 & 1,63 & 1800 & 4371,66 & 2,44 & 2337 \\
\hline & \begin{tabular}{|l}
1 \\
\end{tabular} & 507 & 2,99 & 2,35 & 2,44 & 1800 & 54,29 & 2,45 & 221 \\
\hline & 2 & 101 & 3,00 & 2,35 & 2,35 & 1800 & 73,84 & 2,44 & 464 \\
\hline & 3 & 1528 & 3,00 & 2,35 & 2,34 & 1800 & 79,02 & 2,44 & 692 \\
\hline & 4 & 2037 & 3,00 & 2,35 & 2,21 & 1800 & 94,23 & 2,44 & 918 \\
\hline & 8 & 4073 & 3,00 & 2,35 & 1,92 & 1800 & 128,86 & 2,44 & 1855 \\
\hline & 12 & 6110 & 3,00 & 2,35 & 1,48 & 1800 & 200,33 & 2,44 & 2791 \\
\hline & 16 & 8147 & 3,00 & 2,35 & 1,15 & 1800 & 2578,39 & 2,44 & 3739 \\
\hline & 20 & 10184 & 3,00 & 2,35 & 0,51 & 1800 & 5831,90 & 2,44 & 4668 \\
\hline & 24 & 12218 & 3,00 & 2,35 & 0,26 & 1800 & 30304,09 & 2,44 & 5685 \\
\hline
\end{tabular}

Finally, to confirm the relevance of the approaches proposed, we illustrate in Fig. 3 the profile of the best solution found by MILP in the case of site "7", where 3 batteries are installed, and must be managed over one week. Such a profile is also observed for all other sites for MILP and HEU. The power demand over the time horizon is represented by the blue curve and the power prices by the orange one. The power capacity of each battery installed is represented by the curves in green, purple, and yellow. Firstly, we can observe that batteries can be used in different time periods. In this context, their first use and recharge are performed together, but, in the following, they are used independently from each other. Even during the same battery discharge, there can be different powers, such as in the second use of "Battery-3". Moreover, a battery can be in discharge mode while another one is recharging (e.g., the third use of "Battery-1" and "Battery-3"), and the impact on the maximal number of battery uses imposed (i.e., $N_{b}=3$ ) is observed for 
"Battery-2", that stays a long time in rest mode for this reason. In this example, the energy bill is reduced by $2.70 \%$, confirming the practical relevance of our approaches, and the large variety of battery uses illustrates the need for a decision-making tool.

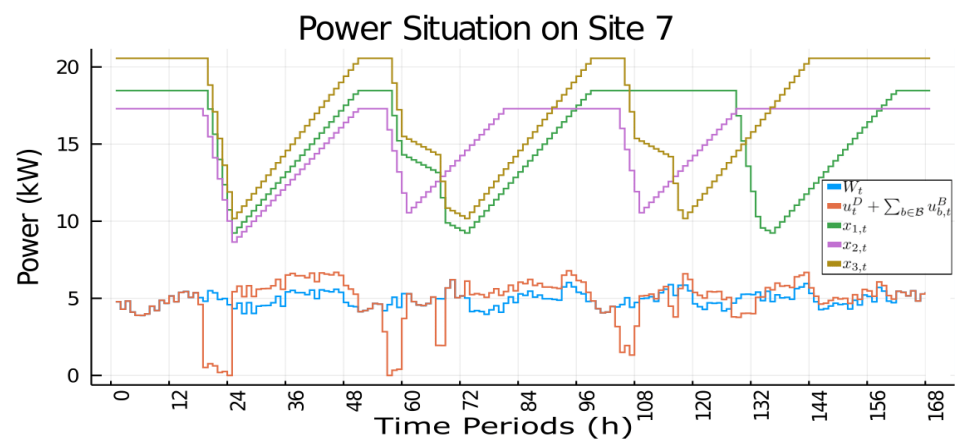

Fig. 3. Illustration of the best solution found by MILP for an OMBSR instance with 3 batteries managed over a week.

Economic Analysis We now focus on the economic aspects of the solutions, and observe a reduction in the energy bill of $2.01 \%$ and $2.33 \%$ on average with MILP and HEU, respectively, confirming that participating in the retail market can generate savings for the company. Furthermore, no substantial gain is observed by increasing the number of batteries installed in a site, which is due to the fact that the sum of the powers of all batteries installed on the site is equivalent to $\bar{W}$, i.e., $\sum_{b \in \mathcal{B}} D_{b}^{\max } \approx \bar{W}$. Indeed, the savings obtained are mainly limited by the maximal number of battery uses and by the fact that the sum of the powers of all batteries installed is equivalent to the average of its power demand. Note that the batteries are primarily installed for backup, and not to participate in the retail market. Moreover, the impact of $N_{b}$ in the savings is observed in both solving methods. The number of times that each battery is used in any solution is exactly $N_{b}$, independently of the solving method used.

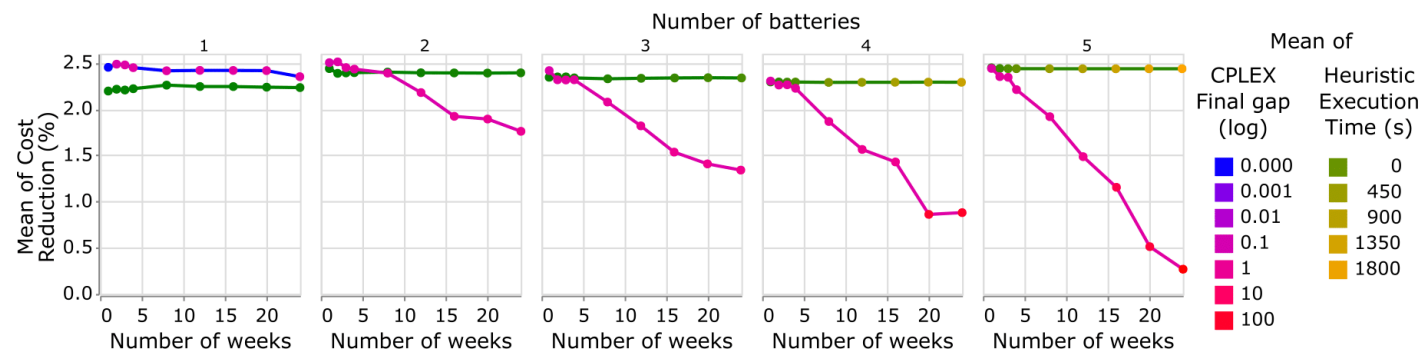

Fig. 4. Results obtained by solving OMBSR with MILP and HEU.

Concerning the MILP results, the savings obtained by the best solution found decreases significantly when the time horizon and the number of batteries installed increase. Such savings decrease from $2.48 \%$ to $0.26 \%$, on average, for large instances. Fig. 4 presents the savings obtained using MILP and HEU, the running times for HEU, and the gap for MILP obtained in our tests. In this figure, only instances for which HEU stops after less than 30 minutes are considered. Concerning MILP, we observe that the savings decrease and the gap increases as the number of batteries installed and the number of time periods increase. As expected, the savings obtained by MILP are larger ( $0.3 \%$ on average) than the ones obtained using HEU for small instances with a single battery. Concerning HEU, the savings obtained are higher compared with the savings obtained with MILP as the number of batteries installed increases. For instances with a single battery installed, savings obtained using HEU are only $0.20 \%$ smaller on average, which seems acceptable for a heuristic that performed 136 times faster, on average, for these instances. Fig. 4 also illustrates the impact of the number of batteries and time periods in the results obtained with HEU. The increase on the number of batteries has a slight impact on the savings, but the raise on the number of time periods does not. 


\section{Conclusion}

This study addresses the use of batteries that are originally installed as a backup in the energy market. In particular, we have considered the problem of optimizing the management of a multi-battery energy storage system in order to reduce the total energy costs, by participating in the demand response mechanism. As a solution method, a mixed-integer linear program is proposed, and any of its optimal solutions provides a strategy for using the batteries so as to reduce the total energy cost. A heuristic is also proposed to solve large instances.

As a result, we observed that using batteries installed for backup in the demand-response mechanism generates savings for the company, hence proving the premise of this study. A series of tests on real instances coming from the French context was performed, in order to analyze the solving approaches as well as the main properties of such instances. We observed in particular that the mixed-integer linear program could achieve an optimality guarantee only for $2.5 \%$ of the instances within the time limit. However, even for instances without such an optimality guarantee, the best solution obtained already generates savings. The savings obtained are significant because the total energy cost can rise to a few million euros per year for telecommunications companies. The number of times that each battery can be used seems to be the parameter that has the greatest impact on those savings. In contrast, no substantial gain was observed by increasing the number of batteries installed. However, the use of multiple batteries is desirable for safety reasons and to increase the lifetime of the batteries. Concerning the heuristic, the results obtained proved its economical relevance, by providing better solutions compared with the best ones obtained by the mixed-integer linear program on large-scale instances.

Concerning the performance of our algorithms, we observed that the numbers of batteries installed and of time periods are the parameters that have the most impact on the solving time. We considered a time limit of 30 minutes for solving each instance, and, in this aspect, the heuristic proved to be computationally efficient (86.4\% of the instances are solved in less than 30 minutes), while we observed that the solving time for the mixed-integer linear program increases fast.

From a research perspective, firstly we aim to extend our study to more realistic scenarios. In the problem addressed, we do not consider the efficiency of a battery nor uncertainty in the power demand and prices. Therefore, taking these aspects into account is a key factor to consider in future research.

\section{Appendix A: (MINLP) Linearizations}

For a product between a binary variable $b_{i}$ and $f_{j} \in\left[0, F^{\text {max }}\right]$ respectively, we can apply the McCormick strategy [20], which amounts to using a new variable $l i n_{-} b f_{i}^{j} \in\left[0, F^{\max }\right]$ to replace this product $b_{i} f_{j}$, together with the following constraints:

$$
\begin{aligned}
& \operatorname{lin}_{-} b f_{i}^{j} \leq b_{i} F^{\max } \\
& \operatorname{lin} \__{-} f_{i}^{j} \leq f_{j} \\
& \operatorname{lin} \__{-} b f_{i}^{j} \geq f_{j}-\left(1-b_{i}\right) F^{\text {max }}
\end{aligned}
$$

The non-linearities of this type in (7) are the products $x_{b, t} z_{b, t}$ (with $x_{b, t} \in\left[0, B^{\max }\right]$ ). We will have the new family of variables $l i n_{-} x z_{b, t}, \forall b \in \mathcal{B}, \forall t \in T$ and the constraints:

$$
\begin{array}{ll}
\text { lin_x } z_{b, t} \leq z_{b, t} B_{t}^{\max } & \forall b \in \mathcal{B}, \forall t \in T \\
\text { lin_x } x z_{b, t} \leq x_{b, t} & \forall b \in \mathcal{B}, \forall t \in T \\
\text { lin_x } x z_{b, t} \geq x_{b, t}-\left(1-z_{b, t}\right) B_{t}^{\max } & \forall b \in \mathcal{B}, \forall t \in T
\end{array}
$$

Furthermore, to linearize $x=\min (a, b)$ for $a, b \in\left[\mathrm{M}^{\prime}, \mathrm{M}\right]$, we introduce a binary variable $y \in\{0,1\}$ such that, if $a>b$, then $y=1$, otherwise, $y=0$. We can then rewrite $x$ as follows: 


$$
\begin{aligned}
& x \leq a, \quad x \leq b \\
& a-b \leq\left(\mathrm{M}-\mathrm{M}^{\prime}\right) y, b-a \leq\left(\mathrm{M}-\mathrm{M}^{\prime}\right)(1-y) \\
& x \geq a-\left(\mathrm{M}-\mathrm{M}^{\prime}\right) y, \quad x \geq b-\left(M-M^{\prime}\right)(1-y)
\end{aligned}
$$

In our case, we have the new family of variable lin_side $_{b, t}$ for all $t$ in $T$ and $b$ in $\mathcal{B}$ to linearize (7). We have $u_{b, t}^{\mathrm{B}}=\left(1-z_{b, t}\right) \min (a, b)$, where $a=B_{b}^{\max }-x_{b, t}$ and $b=\min \left(P_{B_{b}}-W_{t}\right)$. In order to linearize this expression, we have to multiply all the terms $a$ and $b$ in (22) and (24) by $1-z_{b, t}$. Hence, we derive the following constraints, where $M^{\prime}=0$ and $M=\max \left(\mathrm{P}^{\max }, B_{b}^{\max }\right)$ :

$$
\begin{aligned}
& u_{b, t}^{\mathrm{B}} \leq\left(1-z_{b, t}\left(B_{b}^{\max }-x_{b, t}\right), \quad u_{b, t}^{\mathrm{B}} \leq\left(1-z_{b, t}\right) \min \left(P_{B_{b}}, P^{\text {max }}-W_{t}\right)\right. \\
& \left(B_{b}^{\text {max }}-x_{b, t}\right)-\min \left(P_{B_{b}}, P^{\text {max }}-W_{t}\right) \leq \mathrm{M} l i n_{-} \text {side } e_{b, t}, \\
& \min \left(P_{B_{b}}, P^{\text {max }}-W_{t}\right)-\left(B_{b}^{\text {max }}-x_{b, t}\right) \leq \mathrm{M}\left(1-\text { lin_side }_{b, t}\right) \\
& u_{b, t}^{\mathrm{B}} \geq\left(1-z_{b, t}\right)\left(B_{b}^{\text {max }}-x_{b, t}\right)-\mathrm{M}\left(1-z_{b, t}\right) \text { lin_side }_{b, t}, \\
& u_{b, t}^{\mathrm{B}} \geq\left(1-z_{b, t}\right) \min \left(P_{B_{b}}, P^{\text {max }}-W_{t}\right)-\mathrm{M}\left(1-z_{b, t}\right)\left(1-\text { lin_side }_{b, t}\right)
\end{aligned}
$$

Note that, since $u_{b, t}^{\mathrm{B}} \in\left[0, P_{B_{b}}\right]$, Constraints (27) can be replaced by:

$$
\begin{aligned}
& u_{b, t}^{\mathrm{B}} \geq\left(1-z_{b, t}\right)\left(B_{b}^{\max }-x_{b, t}\right)-\mathrm{M} \text { lin_side }_{b, t}, \\
& u_{b, t}^{\mathrm{B}} \geq\left(1-z_{b, t}\right) \min \left(P_{B_{b}}, P^{\text {max }}-W_{t}\right)-\mathrm{M}\left(1-\text { lin_side }_{b, t}\right)
\end{aligned}
$$

Indeed, when $z_{b, t}=0,(27)$ and (28) are equivalent, and, when $z_{b, t}=1,(25)$ together with (28) and $u_{b, t}^{\mathrm{B}} \in\left[0, P_{B_{b}}\right]$ ensure that $u_{b, t}^{\mathrm{B}}=0$.

\section{Appendix B: Heuristic Parameters Tuning}

The arguments for choosing the parameter values are based on the real observed data. Firstly, we observed a daily periodicity in the energy prices and power demand over the time horizon. Figures 5.a and 5.b illustrate such a periodicity for a site over a week. We observe that the energy usually costs more in the afternoon matching with the period of the day that we observe the highest power demand. In addition, the energy tends to cost less during the night following a decreasing power demand. Hence, using batteries in the evening and recharging them during the night appears to be the best moment to reduce the most the total energy cost for the company. Secondly, analyzing the properties of the batteries we observe that they can be used for 9 hours on average and they need about 17 hours on average to be fully recharged. Hence, a complete battery cycle takes 26 hours on average.

Finally, we consider the parameters $\delta=48$ (we have that $26<48<2 * 26=52$ ) and $\delta^{\prime}=24$ due to the daily periodicity observed.

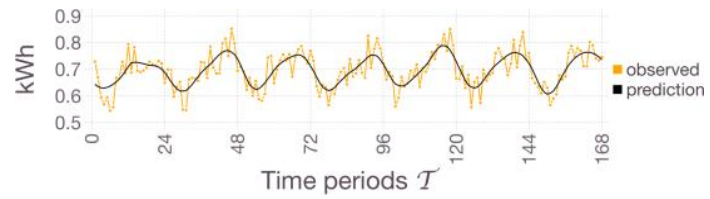

(a)

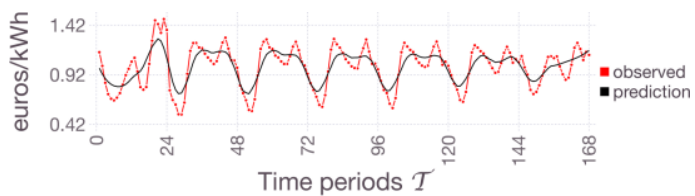

(b)

Fig. 5. (a) Power demand over a week of a site. (b) Power prices over a week of a site.

\section{Appendix C: MWIS-BAT Formulation}

We consider that the interval graph $G=(V, E)$, the corresponding intervals $I$ of $G$, the weights $\omega_{v_{i}}$ and the solutions $S_{i}$ for all $v_{i} \in V$ are given, as well as the values of $N_{b}$ for all $b \in \mathcal{B}$. Then, the following 
auxiliary parameters are considered:

- $\quad N_{b, v_{i}}$ : the number of times that the battery $b$ is used in the solution $S_{i}$.

- $V_{i}$ : set of nodes $v_{i}$ in the interval $i \in I$.

The following variables are considered:

- $\quad x_{v_{i}} \in\{0,1\}$ : equal to 1 if the node $v_{i}$ is taken into the final solution, and to 0 otherwise.

Finally, (MWIS-BAT) can be written as follows:

$$
\begin{aligned}
& \max \sum_{v_{i} \in V_{i}} \omega_{v_{i}} x_{v_{i}} \\
& \text { s.t. } \quad \sum_{v_{i} \in V_{i}} v_{i} \leq 1 \\
& \sum_{v_{i} \in V} x_{v_{i}} N_{b, v_{i}} \leq N_{b} \\
& x_{v_{i}} \in\{0,1\} \\
& \forall i \in I \\
& \forall b \in \mathcal{B} \\
& \forall v_{i} \in V
\end{aligned}
$$

\section{Conflict of Interest}

The authors declare no conflict of interest.

\section{Author Contributions}

All authors conducted the research and had approved the final version. Isaías analyzed the data, conducted the experimental tests, and wrote de paper under supervision of all authors.

\section{References}

[1] Tuan D. The energy web: Concept and challenges to overcome to make large scale renewable and distributed energy resources a true reality. In: Proc. of $20097^{\text {th }}$ IEEE International Conference on Industrial Informatics, 2009: 384-389.

[2] Iordanis K, Vassiliki H, and Leandros T. Optimal energy storage control policies for the smart power grid. In: Proc. of 2011 IEEE International Conference on Smart Grid Communications (Smart-GridComm), 2011: 475-480.

[3] Heinz AK and Krakowski H. Batteries in power supplies for the new telecommunication services. In: Proc. of INTELEC'84 International Telecommunications Energy Conference, 1984: 34-40.

[4] Didier M, Olivier F, and Marc A. Sollan-Dimsol RD. Project, solar and renewable energy in France telecom. In: Proc. of INTELEC 06 - Twenty-Eighth International Telecommunications Energy Conference, Sept, 2006; 1-8.

[5] Bahman D, Roger EB, and Richard DT. Optimal demand-side response to electricity spot prices for storage-type customers. In: Proc. of IEEE Transactions on Power Systems, 1989; 4.3: 897-903.

[6] Jamshid A and Mohammad-Iman A. Demand response in smart electricity grids equipped with renewable energy sources: A review. Renewable and Sustainable Energy Reviews, 2013; 18: 64-72.

[7] Matthew PJ, Amotz Bar-Noy, Ou L, and Yi F. Energy peak shaving with local storage. Sustainable Computing: Informatics and Systems, 2011; 1(3): 177-188.

[8] Aditya M, David I, Prashant S, Jim K, and Ting Z. Smart charge: Cutting the electricity bill in smart homes with energy storage. In: Proc. of the 3rd International Conference on Future Energy Systems: Where Energy, Computing and Communication Meet, 2012: 1-10.

[9] Wael L. "Smart grid-aware radio engineering in 5G mobile networks," $\mathrm{PhD}$ thesis. 2019.

[10] RTE Portal. Data repository. [Online]. Available: www.services-rte.com/fr/telechargez-les-donnees-publiees-par-rte.html.

[11] Anderson H, Alexander B, Shawn C, Annabelle P, and Dragan M. "Look-ahead economic dispatch of micro grids with energy storage, using linear programming. In: Proc. of 2013 1st IEEE Conference on Technologies for Sustainability (SusTech). 2013: $154-161$.

[12] Nicholas G and Pierluigi M. Flexibility in multi-energy communities with electrical and thermal storage: A stochastic, robust approach for multi-service demand response. In: Proc. of IEEE Transactions on SmartGrid, 2017; 10(1): 503-513.

[13] Omowunmi ML. "Optimisation algorithms for energy management in the smart grid". PhD thesis. University of Johannesburg, 2016.

[14] Mousa M, Hamed A, Seyedeh S. Ghazimir-saeid, Hasan U, and Terrence F. Optimal energy management system based on stochastic approach for a home Microgrid with integrated responsive load demand and energy storage. In: Proc. of Sustainable Cities and Society, 2017; 28: 256-264.

[15] Rodrigo M, Roberto M, and Goran S. A MILP model for optimising multi-service portfolios of distributed energy storage. 
Applied Energy, 2015; 137: 554-566.

[16] Hamed B, Babak A, and Ratnesh S. A new control scheme in a multi-battery management system for expanding microgrids. In: Proc. of ISGT2014, 2014: 1-5.

[17] Yuanheng Z, Dongbin Z, Xiangjun L, and Ding W. Control-limited adaptive dynamic programming for multi-battery energy storage systems. In: Proc. of IEEE Transactions on Smart Grid, 2018; 10(4): 4235-4244.

[18] Feilong F, Nengling T, Wentao H, Xiaodong Z, and Chunju F. Distributed equalisation strategy for multi-battery energy storage systems. The Journal of Engineering, 2019;16: 1986-1990.

[19] Isaias FS, Cédric B, Mustapha B, Matthieu C, and Safia KS. Battery energy management of a telecommunications company to participate in the curtailment market and reduce the total energy cost. In: Proc. of 2020 IEEE 8th International Conference on Smart Energy Grid Engineering (SEGE). IEEE. 2020.

[20] Garth P. McCormick. Computability of global solutions to factorable nonconvex programs: Part I-Convex underestimating problems. Mathematical programming, 1976; 10(1): 147-175.

[21] RTE Portal. data.gouv.fr. Electricité: consommation, production, $\mathrm{CO}_{2}$ et échanges. [Online]. Available: www.data.gouv.fr/fr/datasets/electricite-consommation-production-co2-et-echanges/.

Copyright (C) 2022 by the authors. This is an open access article distributed under the Creative Commons Attribution License (CC BY-NC-ND 4.0), which permits use, distribution and reproduction in any medium, provided that the article is properly cited, the use is non-commercial and no modifications or adaptations are made. 